\title{
Detection of Pan drug resistance OXA-48 producing Providencia in an ICU patient for the first time in Nepal
}

\author{
Ranjit Sah ${ }^{1 *}$ D, Shusila Khadka', Gentle Sunder Shrestha², Subhash Acharya ${ }^{2}$, Diptesh Aryal ${ }^{2}$, Pramesh Shrestha², \\ Hari Prasad Kattel ${ }^{1}$, Niranjan Prasad Shah', Bharat Mani Pokhrel ${ }^{1}$, Yogendra Prasad Singh ${ }^{3}$, Basista Rijal ${ }^{1}$ and \\ Hakan Erdem ${ }^{4}$
}

\begin{abstract}
s
Background: Resistance to antimicrobial agents of pathogenic bacteria has become a major problem in routine medical practices. Carbapenem resistance has long been increasing. The production of carbapenem- hydrolysing $\beta$ lactamases (carbapenamases), which include NDM, KPC, OXA-48, IMP-1 and VIM is the most common mechanism.

Case presentation: A 56 years old male presented with fever and mental changes with progressively decreasing sensorium for the last 3 days. He was admitted to Intensive care unit (ICU) with a diagnosis of meningoencephalitis. On day seven, he developed ventilator associated pneumonia due Klebsiella pnemoniae and Acinetobacter baumannii. He was on meropenem, but the isolates were susceptible to colistin, tigecyclin and amikacin solely. Hence, amikacin was started with addition of intravenous and nebulized colistin. Subsequently, vital signs improved with resolution of fever. However, on day 18, he developed fever once again with a drop in blood pressure. Inotropic support was maintained, and echinocandins and tigecycline were added to the regimen. Repeat blood and urine culture grew Providencia species, which were resistant to most of the drugs on phenotypic Kirby-Bauer disk diffusion method and are intrinsically resistant to colistin and tigecycline. Phenotypic detection of ESBL (combined disk method), MBL, KPCs, AmpC and co-producer were tested according to updated CLSI guideline and all were negative. But the Modified Hodges test was found to be positive. Consequenty, OXA-48 drug resistance pattern was brought into action by blank disc method according to A Tsakris et al., which revealed indentation of growth toward both EDTA and EDTA/PBA disk indicating production of OXA-48 carbapenamase. To confirm the resistance pattern we processed the isolated colonies for Xpert Carba-R (Cepheid) assay, which detected blaOXA-48 gene and confirmed the OXA-48 drug resistance pattern. Hence, the infecting organism was not susceptible to any of the antibiotics. The patient was kept under isolation and on 31th day of admission, he died of septic shock.
\end{abstract}

Conclusions: Carbapenamase production along with intrinsic colistin resistance in infecting bacterial pathogens can cause fatal outcomes in the resource limited countries like Nepal where new antibiotic combinations ceftazidime+ Avibactam, or aztreonam +avibactam are not available. Drug resistance patterns including OXA 48 producer should be characterized in all cases by standard phenotypic methods or by Xpert Carba-R assay and larger studies are required to know the exact burden of OXA 48 producer in Nepal.

Keywords: OXA-48, Drug resistance, Providencia species, Nepal

\footnotetext{
* Correspondence: ranjitsah57@gmail.com; ranjitsah@iom.edu.np

'Department of Microbiology, Tribhuvan University Teaching Hospital,

Institute of Medicine, Kathmandu, Nepal

Full list of author information is available at the end of the article
}

(C) The Author(s). 2019 Open Access This article is distributed under the terms of the Creative Commons Attribution 4.0 International License (http://creativecommons.org/licenses/by/4.0/), which permits unrestricted use, distribution, and reproduction in any medium, provided you give appropriate credit to the original author(s) and the source, provide a link to the Creative Commons license, and indicate if changes were made. The Creative Commons Public Domain Dedication waiver (http://creativecommons.org/publicdomain/zero/1.0/) applies to the data made available in this article, unless otherwise stated. 


\section{Background}

The development of antibiotics remains one of the most significant advances in modern medicine. Antibiotics have saved countless lives and continue to be the mainstay of therapy for bacterial infections. The clinical success of the first $\beta$-lactam, penicillin $G$ (benzylpenicillin), prompted the search for and development of additional derivatives. This quest gave rise to the $\beta$-lactam antibiotics in clinical use today (penicillins, narrow- and extended-spectrum cephalosporins, monobactams, and carbapenems) $[1,2]$. Unfortunately, $\beta$-lactamase-mediated resistance to $\beta$ lactam antibiotics emerged as a significant clinical threat to these lifesaving drugs.

There are two globally accepted classification schemes for $\beta$-lactamases, the first one is based on amino-acid sequence classification and the second one is based on functionality. $\beta$-lactamases were divided into four classes (Class A-D) based on their sequence similarity by Ambler in 1980. Classes A, C and D function by the serine ester hydrolysis mechanism, whereas class B $\beta$-lactamases, also known as metallo $\beta$-lactamases, have a zinc ion participating in catalysis [3-5]. The classification scheme by functionality was purposed by Bush et al. in 1995 and was updated in 2009 by Bush-Jacoby group. It takes into account the substrate and inhibitor profiles in an attempt to group the enzymes in ways that can be correlated with their phenotypes in clinical isolates [6]. The updated system includes group 1 (class $C$ ) cephalosporinases; group 2 (classes $\mathrm{A}$ and $\mathrm{D}$ ) broad-spectrum, inhibitorresistant, and extended-spectrum $\beta$-lactamases and serine carbapenamases; and group 3 metallo- $\beta$ lactamases, each of which is also divided into several different subgroups [6]. Awareness is required to all infectious diseases (ID) and general physicians since penicillins, cephalosporins, and carbapenems are included in the preferred treatment regimens for many infectious diseases due to the fact that presence and characteristics of these enzymes play a critical role in the selection of appropriate therapy [6].

Resistance to antimicrobial agents of pathogenic bacteria has become a major problem in current medical practices. Over the last decades, carbapenems have been used as the last-resort drugs in the treatment of serious nosocomial infections caused by multidrugresistant Gram-negative bacteria. However, carbapenem resistance has been increasing and the most common mechanism is the production of carbapeneminactivating $\beta$-lactamases (carbapenamases) [7]. Here, we report an OXA-48 drug resistance pattern observed in blood and urinary isolates of Providencia species in a fatal meningoencephalitis case who subsequently developed ventilator associated pneumonia and urinary tract infection.

\section{Case presentation}

A 56 years old male from Parsa (district) in Nepal presented to emergency department with fever and altered conscious for the last 3 days. His sensorium was progressively worsening, and thus, he was admitted to intensive care unit (ICU) with a diagnosis of meningoencephalitis. The patient was intubated at ICU admission, on day seven he developed ventilator associated pneumonia. Klebsiella pneumoniae and Acinetobacter baumannii were isolated from the sputum sample. He was on meropenem, but the isolates were susceptible to colistin, tigecycline and amikacin solely (beta-lactam antibiotics, fluroquinolones, doxycycline, gentamicin and cotrimoxazole were resistant on phenotypic Kirby-Bauer disk diffusion method). Hence, amikacin was started with addition of intravenous and nebulized colistin. Subsequently, vital signs improved with resolution of fever. However, on day 18, he was febrile once again with drop in blood pressure. Inotropic support was maintained and both echinocandins and tigecycline were added to the regimen.

Although blood and urine cultures grew Providencia species, sputum samples were sterile. Microscopic examination of urine revealed plenty of pus cells, few epithelial cells and no erythrocytes. Patient was at septic shock and he was diagnosed as meningoencephalitis with overlapping ventilator associated pneumonia and urinary tract infection.

The recovered Providencia isolates were resistant to most of the drugs including colistin (intrinsic resistance). Culture of blood and urine revealed gram negative bacilli with non-lactose fermenting colonies on MacConkey and CLED (Cystine Lactose Electrolyte Deficient) agar (Fig. 1)

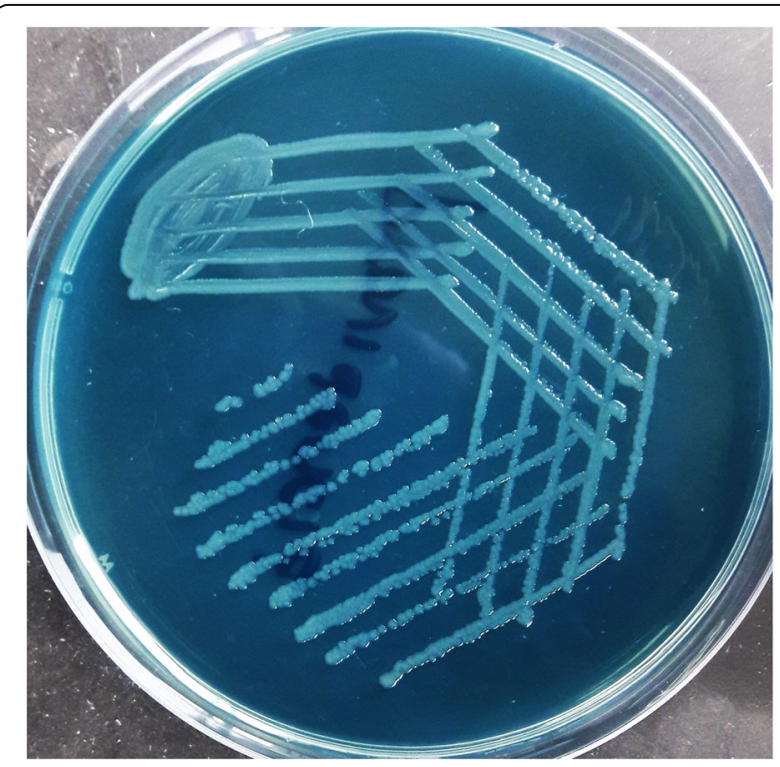

Fig. 1 non-lactose fermenting colonies of Providencia vermicola on CLED (Cystine Lactose Electrolyte Deficient) agar 
respectively. The organism fermented glucose, but could not utilize lactose and sucrose on sugar fermentation test. Sulphite indole motility test revealed motile indole positive organism. Citrate utilization and urea hydrolysis tests were positive. To confirm that the organism belongs to the tribe Proteeae of family Enterobacteriaceae, phenyl pyruvic acid test was performed which was found to be positive (Fig. 2). On the basis of aforementioned phenotypic tests, Providencia species were identified. Antibiotic susceptibility testing was done by Kirby Bauer method according to CLSI (Clinical \& Laboratory Standards Institute) [8] which revealed pan-drug resistant isolate (resistance to almost all drugs available in the laboratory like amoxycillin/ampicillin, ciprofloxacin, levofloxacin, amikacin, gentamicin, meropenem, imipenem, erythromycin, clindamycin, ceftriaxone, ceftazidime, cefoperazone-sulbactam, ampicillin-sulbactum, chloramphenicol, tetracycline, doxycyclin and since Providencia species belongs to tribe Proteeae of family Enterobacteriaceae, they are intrinsically resistant to colistin and tigecycline and therefore drug susceptibility testing is not recommended for this group of organisms).

To characterize the drug resistance pattern, phenotypic detection of ESBL and Modified Hodge test were done according to CLSI guideline [8]. ESBLs were tested by combination disk method in which ceftazidime (CAZ) and ceftriaxone (CTX) alone and in combination with clavulanic acid (CA) $(10 \mu \mathrm{g})$ were used. An increase in zone of inhibition of more than or equal to $5 \mathrm{~mm}$ for either antimicrobial agent in combination with CA versus its zone when tested alone were negative, but Modified Hodges test

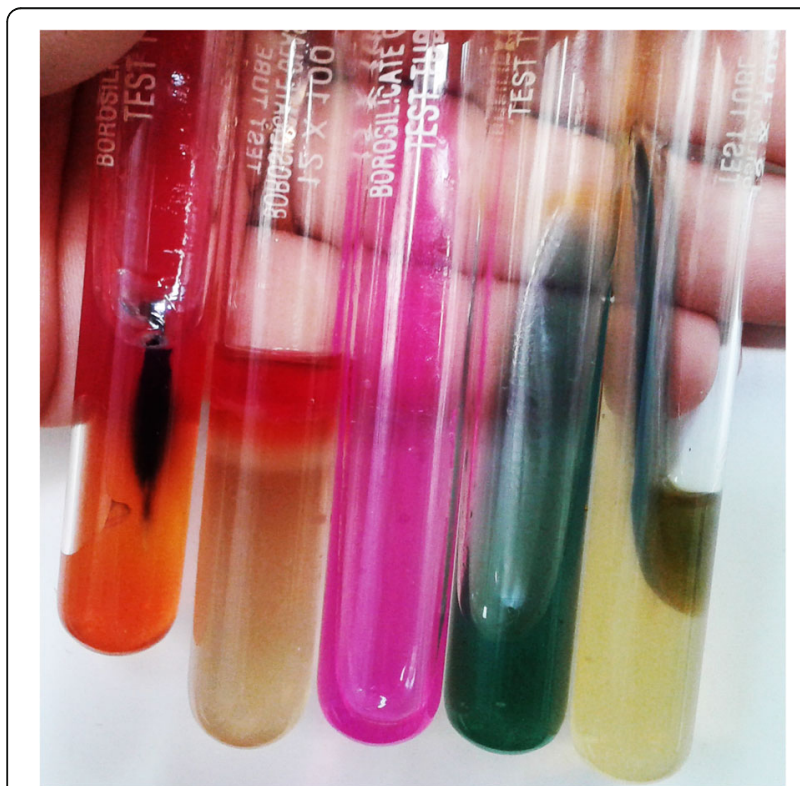

Fig. 2 Biochemical tests showing the organism belonging to Providencia species revealed carbapenamase producing bacteria (Figs. 3 and 4). Subsequently, phenotypic detection of MBL, KPCs, AmpC and co-producer were tested according to CLSI guideline and all were negative. On the background of positive Modified Hodge test and negative ESBL, MBL, KPCs, AmpC and co-producer, OXA-48 drug resistance pattern was brought into action. We used blank disc method for this purpose according to A Tsakris et al. [9] which revealed indentation of growth toward both EDTA and EDTA/PBA disc (Figs.5 and 6) indicating production of OXA-48 carbapenamase. The isolates were transported to India, where they were confirmed as Providencia vermicola by VITEK MS, which use matrix-assisted laser desorption/ionization time-of- flight (MALDI-TOF) technology. Added to that, the resistance pattern of the isolated colonies was processed by Xpert Carba-R (Cepheid) assay, which detect blaOXA48 gene (Figs. 7 and 8) and OXA48 drug resistance pattern was confirmed. The patient was kept under isolation. On 31th day of admission he died of septic shock and lack of other antibiotic option (intrinsic resistance to colistin and unavailability of ceftazidime+ avibactam or aztreonam +avibactam combination in both Nepal and India for testing in laboratory or for systemic use in patient).

\section{Discussion and conclusion}

Among the carbapenamase-producing Enterobacteriaceae species, production of OXA-48 can be suspected when the Modified Hodge Test (MHT) is positive and the inhibitorbased combined disk tests for KPC and MBL production are negative. This phenotypic test (OXA-48 disk test) provided a straightforward, convenient, and accurate means of

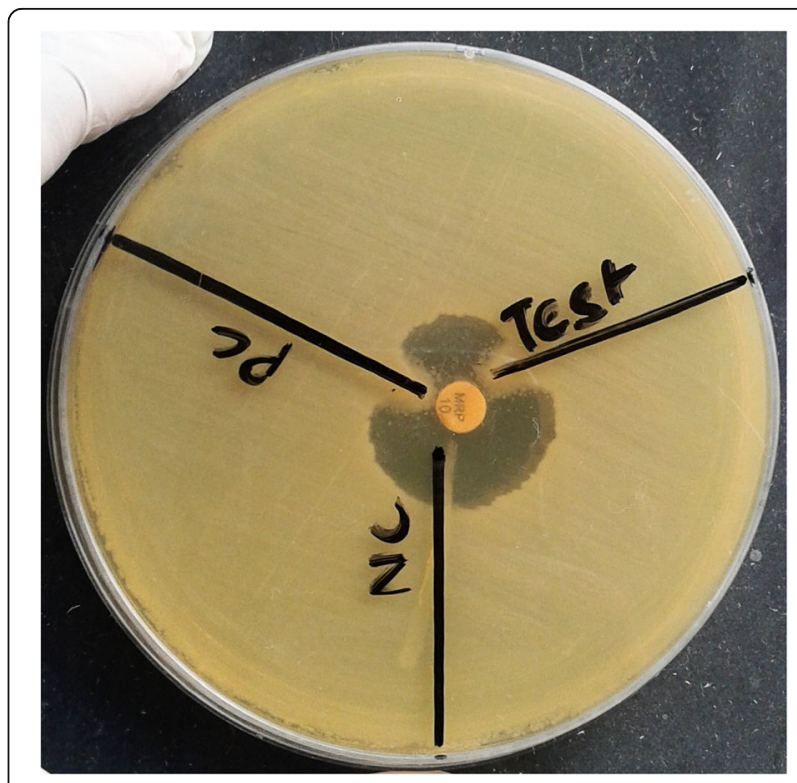

Fig. 3 Modified Hodge Test positive for test organism (Providencia) 


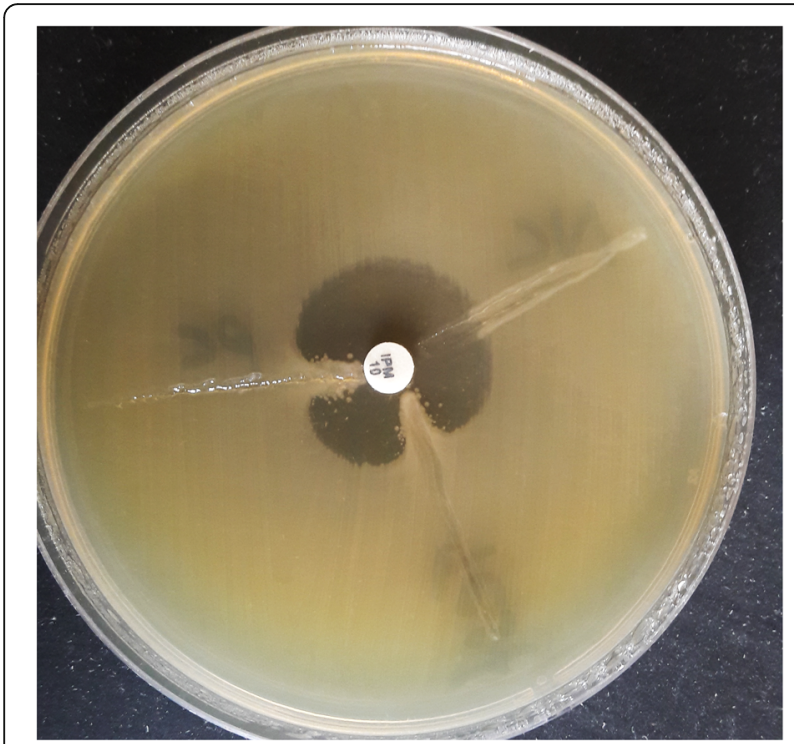

Fig. 4 Modified Hodge Test positive for test organism (Providencia)

detection of OXA-48 carbapenamase in organisms exhibiting reduced susceptibility to carbapenems [9]. In developing countries where genetic confirmation of the isolates and detection of drug resistance gene is not available, phenotypic detection by standard methods and genetic confirmation by Xpert Carba- $\mathrm{R}$ assay is a proper alternative. Cepheid Xpert Carba- $R$ assay is a qualitative, on-demand real-time PCR test for rapid detection and differentiation carbapenamase producing organism by detecting the

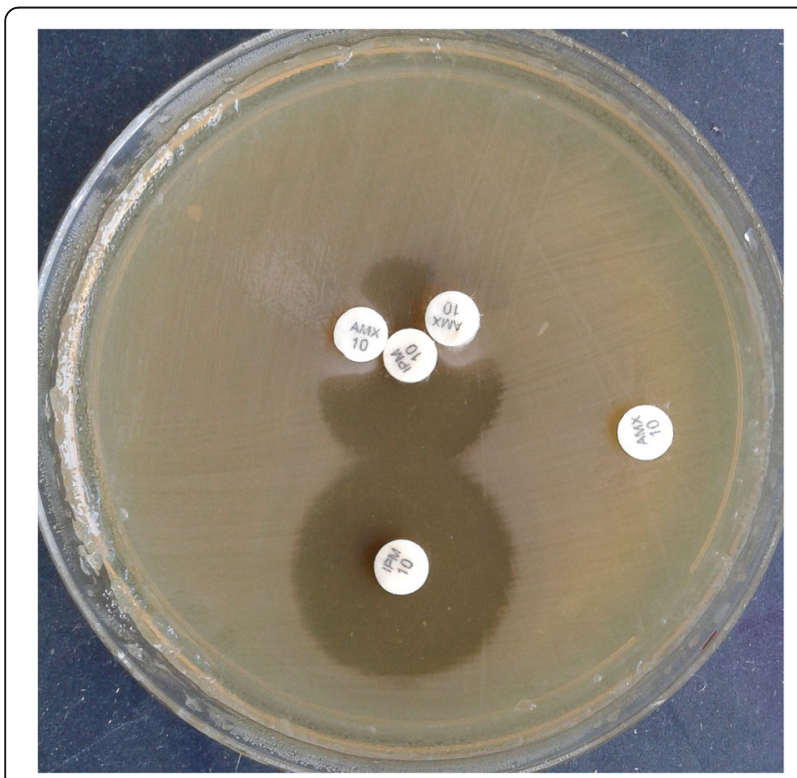

Fig. 5 Detection of OXA-48 by using antibiotic containing disk which is intrinsically resistant to Providencia instead of Blank Disk3. \{E - EDTA-0.1 M $10 \mu \mathrm{l}(292 \mu \mathrm{g}$ of EDTA), P-Phenyl Boronic Acid- $10 \mu \mathrm{L}$ (containing $600 \mu \mathrm{g}$ of PBA)\}3

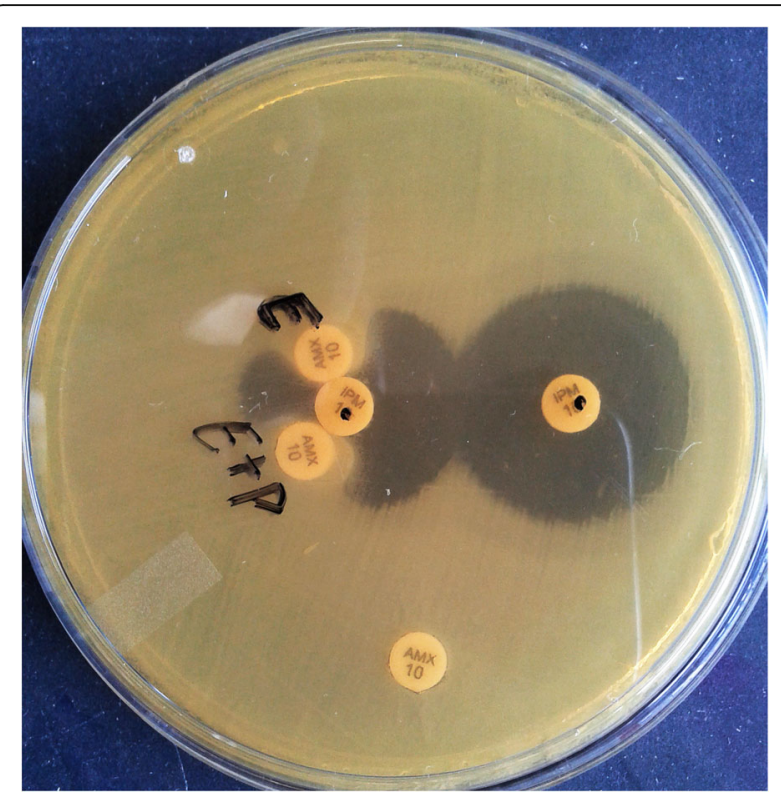

Fig. 6 Detection of OXA-48 by using antibiotic containing disk which is intrinsically resistant to Providencia instead of Blank Disk3. \{E - EDTA-0.1 M $10 \mu \mathrm{l}(292 \mu \mathrm{g}$ of EDTA), P-Phenyl Boronic Acid- $10 \mu \mathrm{L}$ (containing $600 \mu \mathrm{g}$ of PBA)\}3

blaKPC, blaNDM, blaVIM, blaIMP-1 and blaOXA-48 gene sequences from pure colonies encoding the KPC, NDM, VIM, IMP-1, and OXA-48 enzymes respectively and helps clinicians to optimize and direct therapeutic strategy (antibiotic therapy) for patient management [10]. In our case we have first performed phenotypic testing as described by Tsakris et al. [9] and subsequently confirmed OXA-48 enzyme by detecting the blaOXA-48 gene by Xpert Carba- $\mathrm{R}$ assay.

The first identification of OXA-48-producing Enterobacteriaceae was in an isolate in Turkey in 2001 [11, 12]. Shortly thereafter, there was an outbreak of OXA-48producing Klebsiella pneumoniae isolates reported in Istanbul in 2006 [12, 13]. Since its recognition, there have been increasing numbers of reports of OXA-48producing organisms worldwide. Avibactam in combination with either of ceftazidime, cefepime, aztreonam, imipenem, or meropenem can inhibit OXA-48-producing Enterobacteriaceae and are drug of choice [13]. Among them ceftazidime/avibactam has proven to be most successful combination [13]. International Network for Optimal Resistance Monitoring (INFORM) global surveillance study in 2012 to 2015 suggested that the in vitro activity of ceftazidime-avibactam was better against the subset of metallo- $\beta$-lactamase (MBL)-negative, OXA-48- and OXA48-like-positive isolates (99.2 and $100 \%$ susceptible, respectively) [14]. Vasoo et al. also emphasized that ceftazidimeavibactam was active against all KPC-, IMI-, SME-, and most OXA-48 group-producing isolates (93\%), but not to metallo- $\beta$-lactamase producers [15]. 


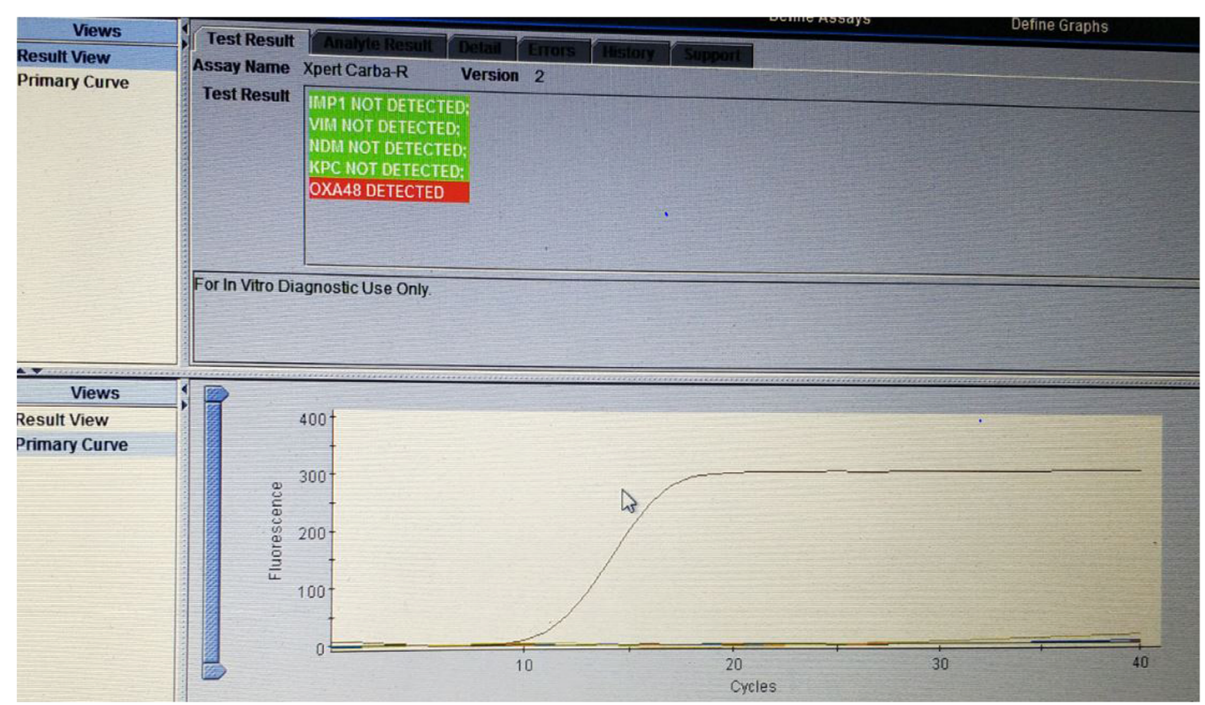

Fig. 7 Cepheid Xpert Carba-R Assay showing detection of blaOXA-48 gene sequences

Sader et al. they analysed in vitro activity of aztreonamavibactam against a large collection (2016) clinical Enterobacteriaceae isolates recovered from patients hospitalized in U.S. medical centers, as well as selected carbapenemase (NDM-like and OXA-48-like)-producing Enterobacteriaceae isolates recovered outside the United States. They found aztreonam-avibactam was very active against KPC, MBL and OXA-48-like producers. Among 57 OXA-48producing isolates obtained from outside the US, two were sensitive with MIC $\leq 0.03 \mu \mathrm{g} / \mathrm{ml}$, two were sensitive with a MIC of $0.06 \mu \mathrm{g} / \mathrm{ml}$, and 17 were sensitive with MIC of
$0.12 \mu \mathrm{g} / \mathrm{ml}, 32$ were sensitive with MIC $0.25 \mu \mathrm{g} / \mathrm{ml}$ and four were sensitive with MIC of $0.5 \mu \mathrm{g} / \mathrm{ml}$. All OXA-48-like producers $(100 \%)$ were sensitive at MIC $0.5 \mu \mathrm{g} / \mathrm{ml}$. They also tested against the 63 strain of MDR Providencia species and eight XDR Providencia species and found sensitivity to aztreonam-avibactam at MIC $0.5 \mu \mathrm{g} / \mathrm{ml}$. They concluded that aztreonam-avibactam may represent a valuable option for treating infections caused by CRE, MDR and XDR Enterobacteriaceae, including OXA-48 and MBL-producing strains [16]. Chew et al. observed that aztreonam-avibactam combination can also be used in dual-carbapenemase-

Assay Information
\begin{tabular}{lll}
$\begin{array}{l}\text { Assay } \\
\text { Xpert Carba- } R\end{array}$ & Assay Version & Assay Type \\
\hline Test Result: & 2 & In Vitro Diagnostic \\
\hline & $\begin{array}{l}\text { MP1 NOT DETECTED; } \\
\text { VIM NOT DETEOTED; }\end{array}$ \\
& NDM NOT DETECTED; \\
& RPC NOT DETECTED; & \\
& OXA48 DETECTED &
\end{tabular}

\begin{tabular}{lllll}
$\begin{array}{l}\text { Analyte Result } \\
\begin{array}{l}\text { Analyte } \\
\text { Name }\end{array}\end{array}$ & Ct & EndPt & $\begin{array}{l}\text { Analyte } \\
\text { Result }\end{array}$ & $\begin{array}{l}\text { Probe } \\
\text { Check } \\
\text { Result }\end{array}$ \\
\hline SPC & 0.0 & 6 & NA & PASS \\
IMP1 & 0.0 & 1 & NEG & PASS \\
VIM & 0.0 & 2 & NEG & PASS \\
NDM & 0.0 & 2 & NEG & PASS \\
KPC & 0.0 & 17 & NEG & PASS \\
OXA48 & 11.3 & 312 & POS & PASS \\
\hline
\end{tabular}

Fig. 8 Cepheid Xpert Carba-R Assay showing detection of blaOXA-48 gene sequences 
producing Enterobacteriaceae to inhibit M $\beta$ Ls (NDM, IMP, or VIM M $\beta$ Ls) with coexistent KPC or OXA-48-like carbapenemases [17].

Hackel et al. tested 1521 isolates of Enterobacteriaceae intrinsically resistant to colistin and found 100\% sensitive to ceftazidime-avibactam [18]. Jayol et al. tested 63 colistin resistant Klebsiella pneumonia isolates, among which 32 were OXA-48 producers and suggested ceftazidime-avibactam is an effective therapeutic option for treating infections caused by colistin resistant and KPC or OXA-48 Klebsiella pneumoniae. In addition to that they also recommend ceftazidimeavibactam and aztreonam combination against colistin resistant and NDM-producing K. pneumoniae [19]. Petrosillo et al. described the use of ceftazidime-avibactam and fosfomycin for colistin resistant $K$. pneumoniae. They also emphasized on soon-to-be commercially available plazomicin and cefiderocol, as novel antimicrobial options and advised to consider the future use of innovative therapeutic strategies in development, including bacteriophages therapy and monoclonal antibodies [20]. OXA 48 producer, which is a global threat has now been detected in Nepal as well. Ceftazidime- avibactam combination or aztreonam -avibactam combination is the drug of choice which is not available in Nepal. Thus, knowledge on the occurrence of the OXA-48 producers may also encourage the pharmaceutical companies and The Ministry of Health to facilitate provision of drug of choice like ceftazidimeavibactam in Nepal.

Since OXA-48 enzyme leads to carbapenem resistance, we are obliged to use colistin and tigecycline due to unavailability of drug of choice in our country. However, some organisms belonging to tribe Proteeae of family Enterobacteriaceae (Proteus, Providencia and Morganella) are intrinsically resistant to colistin and tigecycline. Therefore, organisms which are OXA-48 producers and intrinsically resistant to colistin when infect patients causes high mortality. Use of colistin for colistin susceptible organisms but OXA- producer can help treat the infection, although it may result in adverse effects like renal toxicity. OXA-48 carbapenamase producers are a rising threat to the whole world so that their routine detection is a must. The potential benefits identifying the resistance pattern like OXA producer include initiation of early appropriate therapy in carbapenemase producing isolates.

Through this paper we alarm the official and governmental agencies to focus on the drug resistance mechanisms and to make arrangements for the provision of drugs like ceftazidime+ avibactam or aztreonam + avibactam in Nepal because carbapenamase producing pathogens with intrinsic colistin resistance can be fatal in the resource limited countries where new avibactam-based antibiotic combinations are not available. Protocols should be generated to make this drug available just for the prescription of ID physicians. In addition, developing infectious diseases expert academic programs in the medical institutes of Nepal is needed.

In conclusion, carbapenamase producing bacteria such as MBL, KPC, AmpC and co-producers are frequently detected in our laboratory and in the country. Unfortunately, phenotypic detection of OXA-48 is not routinely practiced. Since, we have detected OXA-48, which results in resistance to almost all antibiotics, drug resistance patterns should be characterized in each case by standard phenotypic method or by Xpert Carba-R assay for appropriate antibiotic selection and better patient care. Also larger studies are required to know the exact burden of OXA 48 producer in Nepal.

\section{Abbreviations \\ AmpC: class C cephalosporinase; CLSI: Clinical and Laboratory Standards Institute; EDTA: Ethylene diamine tetraacetic acid; ESBL: Extended spectrum beta-lactamase; KPC: Klebsiella pneumoniae Carbapenamase; MBL: Metallo- beta-lactamase MHTModified Hodge Test; NDM: New Delhi metallo-beta- lactamase; OXA-48: Oxacillinase-48; PBA: Phenyl Boronic Acid}

\section{Acknowledgements}

We would like to thanks Dr. Mahesh Adhikari, Dr. Neha Shrestha, Prof. Jeevan Bahadur Sherchand, Prof. Keshab Parajuli, Assistant Professor Dr. Sangita Sharma, Dr. Rupendra Thapa, Dr. Samikshya Neupane, Dr. Sanjit Sah, Dr. Shyam Sah and Dr. Ranjana Sah for their constant support and guidance.

\section{Authors' contributions}

RS made the diagnosis, RS, SK, GSS, SA, DA, PS, HPK, NPS, BMP, YPS and BR designed the manuscript, reviewed the literature and prepared the article for submission. GSS, SA, DA, PS, HPK, NPS, BMP, YPS and BR helped for literature review, gave concept of research paper and critically reviewed the manuscript. HE helps to review the literature, revise the article and improved the content of the article. All authors read and approved for the final manuscript. The manuscript has not been previously published nor is not being considered for publication elsewhere.

\section{Funding}

"No funding was received".

\section{Availability of data and materials}

Data generated or analyzed during this study are included in this published article and remaining are available from the corresponding author on reasonable request.

\section{Ethics approval and consent to participate}

There is no need for ethical approval for a case report according to the local ethical guidelines.

\section{Consent for publication}

Written informed consent was taken from the patient's wife for publication of this Case Report and any accompanying images.

\section{Competing interests}

The authors declare that they have no competing interests.

\section{Author details}

'Department of Microbiology, Tribhuvan University Teaching Hospital, Institute of Medicine, Kathmandu, Nepal. ${ }^{2}$ Department of Anesthesiology (ICU), Tribhuvan University Teaching Hospital, Institute of Medicine, Kathmandu, Nepal. ${ }^{3}$ Research Department, Institute of Medicine, Kathmandu, Nepal. ${ }^{4}$ IDI-IRI, Ankara, Turkey. 
Received: 10 May 2019 Accepted: 21 September 2019

Published online: 15 October 2019

\section{References}

1. Drawz SM, Robert A. Bonomo Three Decades of b-Lactamase Inhibitors. Clin Microbiol Rev. 2010;23(1):160

2. Shaikh S, Fatima J, Shakil S, Rizvi SM, Kamal MA. Antibiotic resistance and extended spectrum beta-lactamases: types, epidemiology and treatment Saudi J Biol Sci. 2015;22(1):90-101.

3. Dever LA, Dermody TS. Mechanisms of bacterial resistance to antibiotics Arch Intern Med. 1991 May:151(5):886-95.

4. Munita JM, Arias CA. Mechanisms of Antibiotic Resistance. Microbiol Spectr. 2016 Apr:4:2. https://doi.org/10.1128/microbiolspec.VMBF-0016-2015.

5. Peterson E, Kaur P. Antibiotic resistance mechanisms in Bacteria: relationships between resistance determinants of antibiotic producers, environmental Bacteria, and clinical pathogens. Front Microbiol. 2018;9:1-21 https://doi.org/10.3389/fmicb.2018.02928.

6. Bush K, Jacoby GA. Updated functional classification of beta-lactamases. Antimicrob Agents Chemother. 2010;54(3):969-76.

7. Studentova $V$, et al. Detection of OXA-48-type carbapenamaseproducing Enterobacteriaceae in diagnostic laboratories can be enhanced by addition of bicarbonates to cultivation media or reaction buffers. PMC. 2015;60(2):119-29.

8. Performance Standards for Antimicrobial Susceptibility Testing; Twenty-Sixth Informational Supplement. Wayne, Pennsylvania, USA: CLSI; 2016.

9. Tsakris A, et al. Evaluation of a New Phenotypic OXA-48 Disk Test for Differentiation of OXA-48 Carbapenamase-Producing Enterobacteriaceae Clinical Isolates. JCM. 2015:53:4.

10. Tato M, Ruiz-Garbajosa P, Traczewski M, Dodgson A, McEwan A, Humphries $R$, et al. Multisite evaluation of Cepheid Xpert Carba-R assay for detection of Carbapenamase-producing organisms in rectal swabs. J Clin Microbiol. 2016; 54(7):1814-9.

11. Poirel L, Héritier C, Tolün V, Nordmann P. Emergence of oxacillinase-mediated resistance to imipenem in Klebsiella pneumoniae. Antimicrob Agents Chemother. 2004:48(1):15-22. https://doi.org/10.1128/aac.48.1.15-22.2004

12. Carrër A, Poirel L, Eraksoy H, Cagatay AA, Badur S, Nordmann P. Spread of OXA-48-positive carbapenem-resistant Klebsiella pneumoniae isolates in Istanbul, Turkey. Antimicrob Agents Chemother. 2008;52(8):2950-4. https:// doi.org/10.1128/AAC.01672-07.

13. Stewart A, Harris P, Henderson A, Paterson D. Treatment of Infections by OXA48-Producing Enterobacteriaceae. Antimicrob Agents Chemother. 2018:62:11.

14. Kazmierczak KM, Bradford PA, Stone GG, de Jonge BLM, Sahm DF. In Vitro Activity of Ceftazidime-Avibactam and Aztreonam-Avibactam against OXA48-Carrying Enterobacteriaceae Isolated as Part of the International Network for Optimal Resistance Monitoring (INFORM) Global Surveillance Program from 2012 to 2015. Antimicrob agents Chemother. 2018;62(12):e00592e00518 Published 2018 Nov 26. doi:https://doi.org/10.1128/AAC.00592-18.

15. Vasoo S, Cunningham SA, Cole NC, et al. In vitro activities of CeftazidimeAvibactam, Aztreonam-Avibactam, and a panel of older and contemporary antimicrobial agents against Carbapenemase-producing gram-negative bacilli. Antimicrob Agents Chemother. 2015;59(12):7842-6. https://doi.org/ 10.1128/AAC.02019-15.

16. Sader HS, Mendes RE, Pfaller MA, Shortridge D, Flamm RK, Castanheira M. Antimicrobial Activities of Aztreonam-Avibactam and Comparator Agents against Contemporary (2016) Clinical Enterobacteriaceae Isolates. Antimicrob Agents Chemother 2017;62(1):e01856-17 Published 2017 Dec 21. doi:https://doi.org/10.1128/AAC.01856-17.

17. Chew KL, Tay MKL, Cheng B, Lin RTP, Octavia S, Teo JWP. AztreonamAvibactam Combination Restores Susceptibility of Aztreonam in DualCarbapenemase-Producing Enterobacteriaceae. Antimicrob Agents Chemother. 2018;62(8):e00414-e00418. Published 2018 Jul 27. doi:https:// doi.org/10.1128/AAC.00414-18

18. M. Hackel, S. Bouchillon, R. Badal, W.W. Nichols. Activity of Ceftazidime-Avibactam and Comparators against Colistin NonSusceptible Enterobacteriaceae and Pseudomonas aeruginosa Isolated in 2012: The International Network For Optimal Resistance Monitoring (INFORM) Study. 24 ${ }^{\text {th }}$ ECCMID Congress, Barcelona, Spain, 10-13 May 2014, eP437.

19. Jayol A, Nordmann P, Poirel L, Dubois V. Ceftazidime/avibactam alone or in combination with aztreonam against colistin-resistant and carbapenemase-producing Klebsiella pneumoniae. J Antimicrob Chemother. 2018:73(2):542-4. https://doi.org/10.1093/jac/dkx393.

20. Petrosillo N, Taglietti F, Granata G. Treatment Options for Colistin Resistant Klebsiella pneumoniae: Present and Future. J Clin Med. 2019 Jun 28;8:7. https://doi.org/10.3390/jcm8070934.

\section{Publisher's Note}

Springer Nature remains neutral with regard to jurisdictional claims in published maps and institutional affiliations.
Ready to submit your research? Choose BMC and benefit from:

- fast, convenient online submission

- thorough peer review by experienced researchers in your field

- rapid publication on acceptance

- support for research data, including large and complex data types

- gold Open Access which fosters wider collaboration and increased citations

- maximum visibility for your research: over $100 \mathrm{M}$ website views per year

At BMC, research is always in progress.

Learn more biomedcentral.com/submissions 